\title{
Sling bulbouretral. Experiencia del servicio
}

\author{
M. Cerqueira, L. Xambre, V. Silva, R. Santos, R. Lages, R. Prisco, F. Carreira \\ Servicio de Urología del Hospital Pedro Hispano. Matosinhos. Portugal.
}

Actas Urol Esp 2005; 29 (4): 401-407

\section{RESUMEN}

SLING BULBOURETRAL - EXPERIENCIA DEL SERVICIO

Objetivos: En el artículo los autores presentan los resultados clínicos verificados en los pacientes portadores de incontinencia urinaria de stress, tras el uso del sling bulbouretral de fijación en el hueso púbico (Invance ${ }^{\circledR}$ ).

Material y métodos: Entre Junio de 2003 y Diciembre de 2003, fueron implantados 10 slings en hombres con edades comprendidas entre los 60 y los 83 años (media 72,6 años), los cuales presentaban incontinencia tras cirugía prostática (prostatectomía radical retropúbica, prostatectomía radical perineal, cistoprostatectomía radical con neovejiga Camey II, resección transuretral o adenomectomía prostática transvesical).

Resultados: Tras seguimiento de 9 meses ( 7 a 13 meses), 8 pacientes (80 \%) están continentes (no necesitando utilizar compresas) y $2(20 \%)$ presentan pérdidas mínimas al realizar algún esfuerzo (con la necesidad de una a dos compresas diarias). Todos ellos están satisfechos con el resultado de la cirugía realizada. Dos pacientes presentaron dolor perinanal que respondió con el uso de paracetamol. No se verificó ningún caso de hematoma perinanal, infección, rechazo o erosión uretral.

Conclusión: El sling bulbouretral con fijación ósea, es un procedimiento quirúrgico poco invasivo, de fácil ejecución técnica, con tasas de continencia elevadas y asociado a baja morbilidad. A pesar de los resultados positivos presentados por nuestro equipo, que pueden resultar un estímulo para la continuación de la técnica, consideramos que será necesario un mayor seguimiento y una mayor experiencia global para que esta técnica quirúrgica se afirme y se transforme en una alternativa a la utilización del esfinter artificial.

Palabras clave: Cáncer de próstata. Incontinencia. Cabestrillo masculino.

\section{ABSTRACT \\ BULBOURETHRAL SLING. THE EXPERIENCE OF OUR SERVICE}

Objectives: The authors present the clinic results obtained with the bulbourethral sling application with pubic bone anchorage (Invance ${ }^{\circledR}$ ) in patients with stress urinary incontinence.

Material and methods: From July to December 2003, 10 slings were implanted in men between the 60's and the 83 years old (average 72,6 years), whose incontinence appeared after prostatic surgery (retro pubic radical prostatectomy, perineal radical prostatectomy, radical cystoprostatectomy with Camey II neobladder, transurethral resection, transvesical prostate adenomectomy).

Results: After 9 months follow-up (3 to 7 months), 8 patients (80\%) are continent (without need of using any pad) and 2 (20\%) show minimum leakage with effort (need of 1 to 2 daily pads). All are satisfied with the surgery result. Two patients referred perineal pain, which was solved with Paracetamol. There was no case of perineal haematoma, infection, rejection or urethral erosion.

Conclusion: The bulbourethral sling with bone anchorage is a rather invasive procedure, of easy technical execution, with high continence rates and associated to low morbidity. Although the presented results been an incentive to the technique prolongation, it would be necessary a higher tracking: and higher global experience in order that this sling affirm and transform itself in an alternative to the artificial sphincter in selected cases. 
La incontinencia urinaria de stress surge en un número significativo de pacientes sometidos a cirugia prostática (cerca de 3 a $60 \%$ de los pacientes sometidos a prostatectomía radical y cerca de $1 \%$ de los sometidos a adenomectomía o resección prostática transuretral) ${ }^{1}$.

En la literatura médica están descritas varias opciones terapéuticas para aliviar o resolver la incontinencia urinaria, tales como el uso de colectores externos, clamps peneanos, sondas Foley, inyección periuretral de colágeno (u otros materiales), esfinter artificial y slings bulbouretrales. Las técnicas de slings han adquirido importancia actual en el tratamiento de la incontinencia de stress, por su simplicidad técnica y su gran eficacia terapéutica.

Los autores presentan los resultados clínicos obtenidos con la aplicación del sling bulbouretral con fijación en el hueso púbico (Invance®).

\section{MATERIAL Y MÉTODOS}

De Junio de 2003 a Diciembre de 2003 fueron implantados 10 slings en hombres de 60 a 83 años (medía 72,6 años).

A todos los pacientes se les realizó una historia clínica detallada con examen físico completo; posteriormente todos ellos fueron sometidos a un estudio urodinámico y cistoscopia para evaluación de la incontinencia.

Cinco pacientes habían sido sometidos a prostatectomía radical retropúbica, dos a prostatectomía radical perineal, uno a adenomectomía transvesical, uno a resección prostática transuretral y otro a cistoprostatectomía radical con neovejiga Camey II.

Tres de los pacientes realizaron radioterapia externa y cinco fueron sometidos a cervicotomía endoscópica por estenosis del neo-colon. Dos pacientes fueron sometidos a inyección periuretral de prótesis química (Deflux®) no presentando mejoría de la incontinencia. Todos ellos presentaban incontinencia a partir de la cirugía, no revelando mejorías con el paso del tiempo. El período de tiempo entre la cirugía que motivó la incontinencia y la colocación del sling osciló entre 9 y 62 meses (medía 27,7 meses) (Tabla 1).

En el estudio urodinámico se verifico que todos los pacientes tenían sensibilidad vesical conservada (excepto en el paciente con neo-veji-
Tabla 1

Casuística y datos demográficos

Pacientes: 10

Prostatectomía radical: 7

- 5 tras cervicotomía endoscópica

- 3 tras radioterapia externa

Adenomectomia prostática transvesical: 1

Resección transuretral de la próstata: 1

Cistoprostatectomía radical con neo-vejiga tipo Camey II: 1

Edad medía 72,6 años (60 a 83 años)

Tiempo tras la cirugía 27,7 meses (9 a 62 meses)

Tratamientos previos (Deflux®): 2 pacientes

ga), siendo la capacidad vesical media de $340 \mathrm{ml}$ (230-464 ml), la acomodación vesical era normal en todos los pacientes y ninguno presentaba inestabilidad vesical. Todos ellos presentaban Valsalva liak point pressure (VLPP) para bajas presiones.

A todos los pacientes se les administró $2 \mathrm{~g}$ de ceftriaxone antes de la cirugía. Fueron colocados en posición de litotomía, procediéndose después a la colocación de una cistostomía percutánea suprapúbica, excepto en el paciente con neovejiga, en el que se optó por la colocación de una sonda vesical. En todos los pacientes fue utilizado un kit quirúrgico que disponía de todo el material necesario para el procedimiento (tornillos de titanio con hilos de Prolene ${ }^{\circledR}$ acoplados, introductor y red sintética) (Fig. 1).

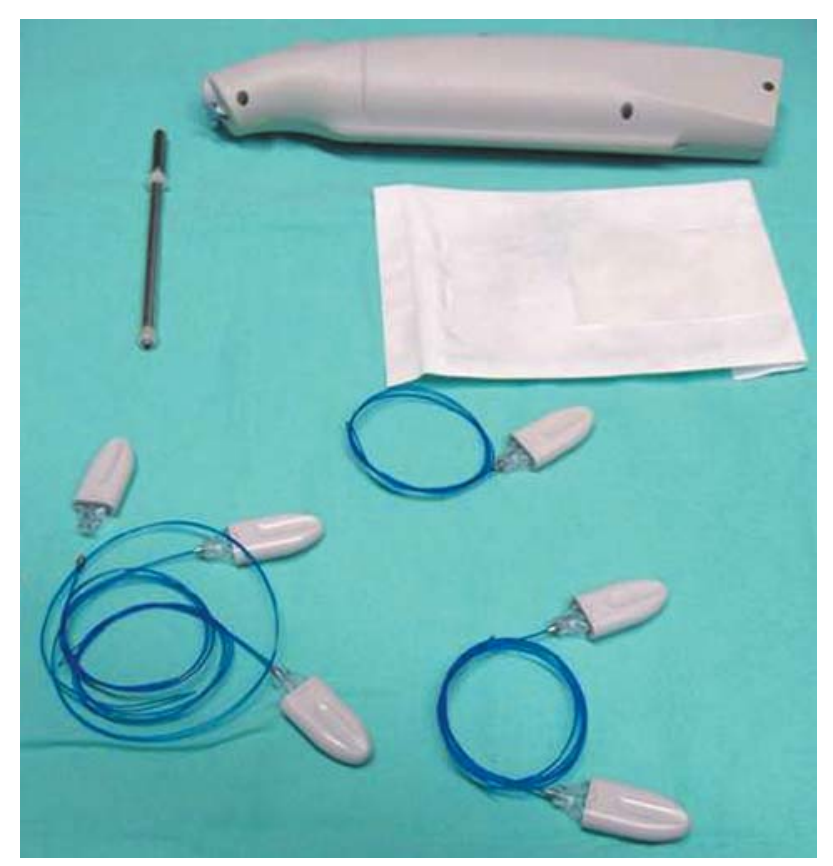

FIGURA 1. Kit de material. 
La técnica quirúrgica se inicia con una incisión longitudinal perianal de unos $5 \mathrm{~cm}$, la cual permite exponer el músculo bulbocavernoso y lateralmente los ramos isquiopúbicos, siendo importante obtener una clara visualización de la superficie ósea (periostio) del borde medíal de estos ramos (Fig. 2). Sobre esta superficie son colocados 4 tornillos de titanio con hilo de Prolene ${ }^{\circledR}$ acoplado, un par junto a la sínfisis púbica y otro par más o menos $3 \mathrm{~cm}$ infero-lateralmente. Se coloca la red sintética sobre el músculo bulbocavernoso y se asegura con los hilos de Prolene ${ }^{\circledR}$. Posteriormente se ajusta la red de modo que proporcione el grado de tensión deseada sobre la uretra bulbar y estructuras adyacentes. Por fin, se cierra la incisión con hilo sintético reabsorvíble (Fig. 3).

En los pacientes con cistostomía se realizó clampaje a las 24 horas y se retiró a las 48 horas después de ser verificada la ausencia de residuo post-miccional. El paciente con neo-vejiga mantuvo la sonda vesical por 48 horas.

\section{RESULTADOS}

Después de $24 \mathrm{~h}$ de post-operatorio, la cistostomía fue clampada y todos los pacientes presentaron diuresis espontánea con residuos vesicales nulos ( 3 mediciones sucesivas) por lo que se tomó la decisión de retirar la cistostomia.

En el paciente con neo-vejiga se retiro la sonda vesical a las 48 horas, siendo posible la micción con el recurso a la maniobra de Credé, no verificándose ninguna dificultad para la micción y observándose volúmenes post-miccionales residuales inferiores a $20 \mathrm{ml}$. Al enfermo le fue explicada la necesidad de realizar micciones regulares ( $3 / 3$ horas) con el recurso a las maniobras de compresión, recibiendo el alta al $5^{\circ}$ día de

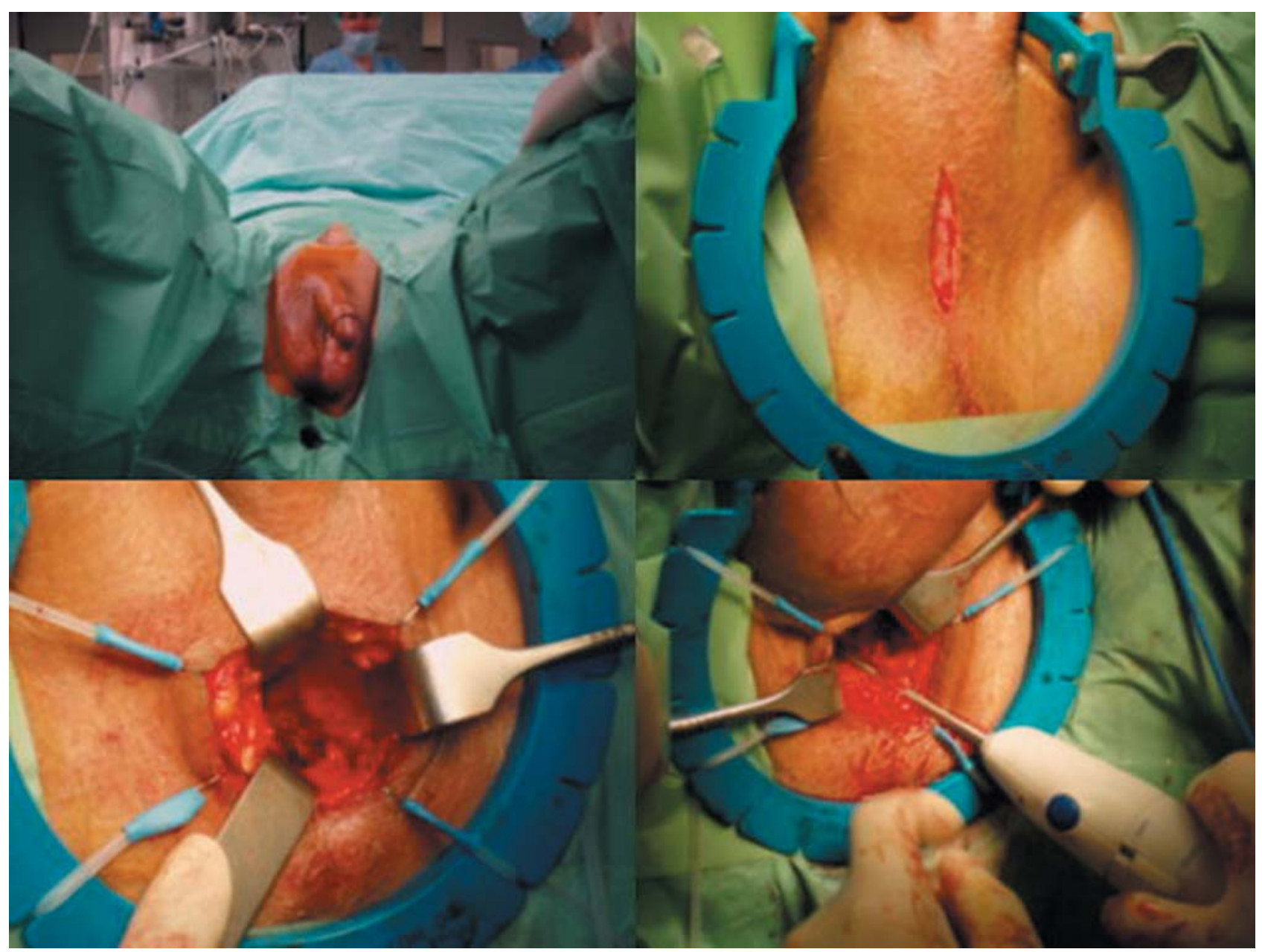

FIGURA 2. Técnica quirúrgica. 


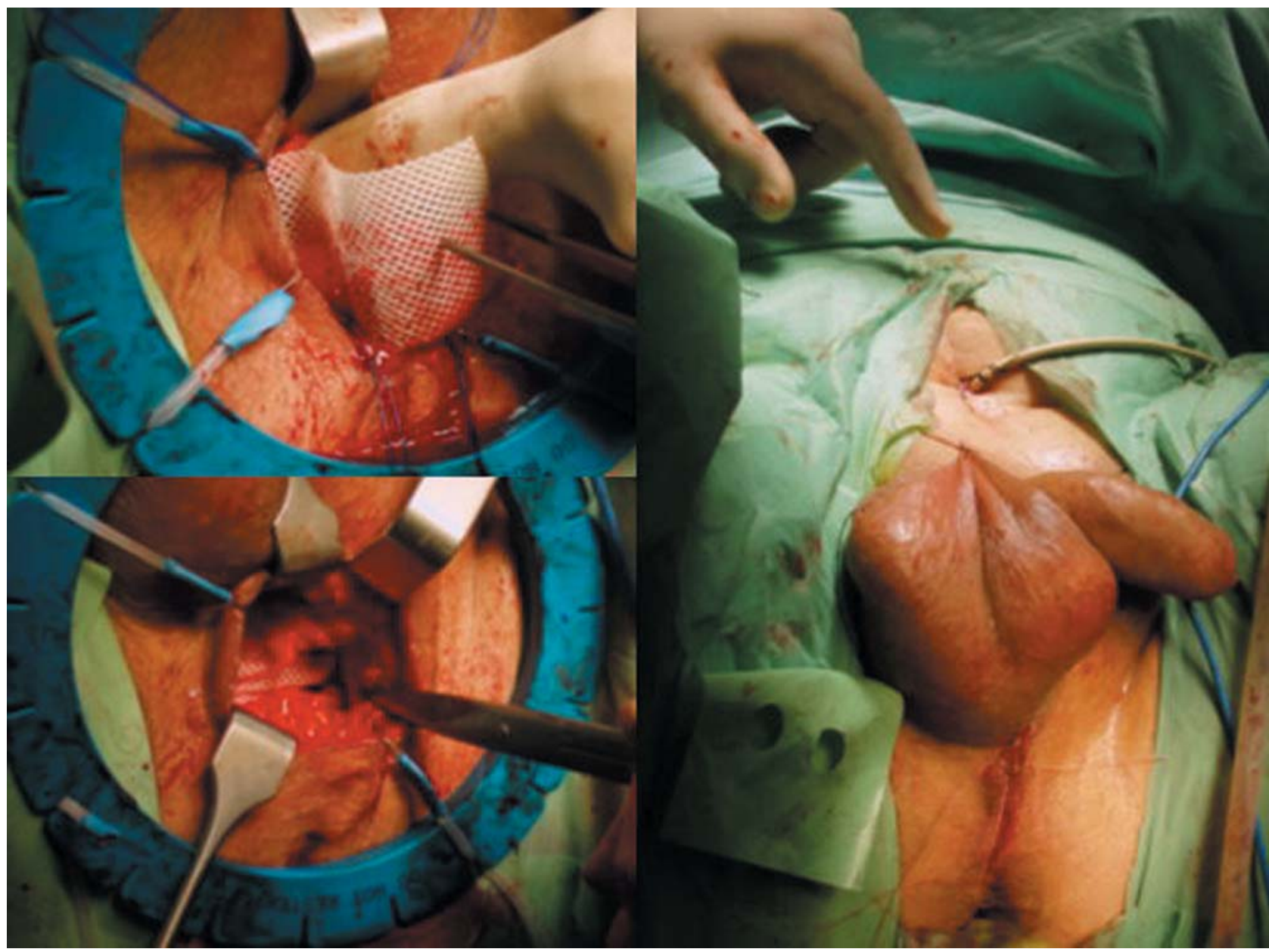

FIGURA 3. Técnica quirúrgica (cont.)

post-operatorio, mientras que los restantes pacientes lo hicieron al $3^{\circ}$ día de post-operatorio. El tiempo de ingreso medio fue de 4,2 días (4 a 6 días). Las perdidas sanguíneas en el intra y postoperatorio fueron prácticamente nulas, siendo el tiempo quirúrgico medio de 45 minutos ( 35 a 60 minutos).

$\mathrm{El}$ seguimiento fue realizado en la consulta externa, al $1^{\circ}, 3^{\circ}, 6^{\circ}$ y $12^{\circ}$ mes, tras cirugía; el seguimiento medio fue de 9 meses ( 7 a 13 meses).

Todos los pacientes recibieron el alta siendo continentes ( $\sin$ necesidad de compresas) y permanecian continentes un mes después de la cirugía. Al tercer mes, dos pacientes (20\%) presentaban ligeras perdidas involuntarias asociadas al stress (necesidad de una a dos compresas diarias), encontrándose los restantes continentes. Al sexto mes, los pacientes mantenían el mismo estado de continencia urinaria observado en la consulta previa. Dos pacientes alcanzaron y superaron el primer año de seguimiento manteniéndose continentes (Tabla 2). En términos de complicaciones, dos pacientes $(20 \%)$ relataron dolor perianal en las primeras 48 horas que se calmó con la toma de paracetamol. No se verificó ninguna otra morbilidad o complicación, tales

\section{Tabla 2}

Resultados

Tiempo operatorio medio: 45 minutos (35 a 60 minutos) Perdidas séricas: prácticamente nulas Re-intervención: 0

Tiempo de internamiento: 4,2 días (4 a 6 días) Seguimiento medio: 9 meses (7 a 13 meses) Continencia:

- Secos: 8 pacientes (80\%)

- Mejorados: 2 pacientes (20\%)

Satisfacción: $100 \%$ 
como: hematomas, urgencia miccional de novo, infecciones, rechazo o erosión uretral. Todos los pacientes se muestran satisfechos con el resultado obtenido con la cirugía, incluso los dos pacientes con ligeras pérdidas, no las valoran por no considerarlas socialmente incómodas (Tabla 3).

Tabla 3

Complicaciones

\begin{tabular}{lc}
\hline Complicación mayor & 0 \\
Erosión & 0 \\
Complicación minor & $2(20 \%)$ \\
Hematoma perineal & 0 \\
Osteíte púbica & 0 \\
Hipostesia perineal / genital & 0 \\
Dolor perineal / genital & 2 \\
Imperiosidad miccional "de novo" & 0 \\
Mortalidad & 0 \\
Total & $2(20 \%)$ \\
\hline
\end{tabular}

\section{DISCUSIÓN}

La incontinencia urinaria post-prostatectomía es una complicación temida, dado el deterioro que provoca en la calidad de vida del paciente.

Existen múltiples alternativas terapéuticas, algunos con resultados clínicos insatisfactorios. Los ejercicios pélvicos y las técnicas de "biofeedback", deben ser las primeras opciones terapéuticas, sobre todo durante el primero trimestre de post-operatorio y en los casos de pérdidas ligeras. Estas técnicas lamentablemente tienen tasas de recidiva elevada en especial tras la suspensión de los ejercicios. En los pacientes con incontinencia superior a un año de evolución o con perdidas que van de moderadas a graves, resulta poco probable que estas técnicas puedan tener éxito.

Las sondas vesicales permiten evitar la incontinencia, pero la permanencia prolongada de un material intra-vesical extraño, crea condiciones que favorecen la aparición de infecciones urinarias, formación de cálculos y la transformación metaplásica de la mucosa vesical. La colocación de colectores externos es una solución que implica algunas dificultades, pues la colocación del preservativo al pénis no resulta siempre posible y obliga al uso de sacos colectores. Otros métodos mínimamente invasivos son capaces de ofrecer beneficios en el tratamiento de la incontinencia urinaria, pero no están exentos de desventajas; los clamps peneanos están asociados a lesiones cutáneas, resultado de su uso regular ${ }^{2}$.

Los resultados de la inyección transuretral de una prótesis química fueron prometedores inicialmente; estudios posteriores mostraron inequívocamente que esta conclusión no era favorable en todos los casos. Los mejores resultados fueron obtenidos en pacientes sometidos a adenomectomia $(62,5 \%)$, siendo los resultados significativamente peores en pacientes sometidos a prostatectomia radical $(8 \text { a } 35 \%)^{1,2}$. La necesidad de múltiples inyecciones para obtener continencia, constituye un importante encarecimiento del tratamiento. Algunos autores consideran incluso, que estos gastos globalmente, son más elevados que los ocasionados con la colocación del esfínter artificial. Este es un procedimiento que debe ser reservado para pacientes con incontinencia ligera y conscientes de la eventual necesidad de de tratamientos múltiples.

La implantación de un esfinter urinario artificial obtiene tasas de curación entre 60 y los $80 \%$, pero con una morbilidad elevada. Posteriores revisiones y la necesidad de retirar el esfinter (por infecciones, erosión de la uretra, etc.) son frecuentes llegando a alcanzar tasas entre 17 a $57 \%$ y de 14 a $17 \%$ a los 5 años, respectivamente $^{2}$. A pesar de ser considerado el tratamiento de elección para la incontinencia de stress en el hombre, no es un tratamiento posible de aplicar a todos los pacientes. El funcionamiento del esfinter urinario artificial, requiere obligatoriamente la colaboración activa del paciente, el cual tiene que tener una capacidad física e intelectual que permita la adecuada manipulación del dispositivo. Como es evidente, esto no resulta siempre posible, ya que muchos pacientes poseen limitaciones fisicas o intelectuales.

Las técnicas de sling, disfrutan de un largo historial en el tratamiento de la incontinencia urinaria femenina, donde se registran tasas de éxito próximas de $90 \%$. Esta técnica se presentan actualmente como una solución alternativa para la incontinencia masculina ${ }^{3}$. En todas estas técnicas se procura restaurar los mecanismos fisiológicos de la micción, y en este sentido, se resuelve uno de los problemas que 
tiene el esfinter urinario artificial, dado que no depende de la colaboración del paciente, y pueden ser implantados en hombres con limitaciones fisicas y/o intelectuales.

En la década de 70, Kaufman describió algunas técnicas para el tratamiento de la incontinencia masculina, utilizando la compresión de los tejidos peri-uretrales, obtenidos con el encruzamiento de los crura (Kaufman I), con prótesis de politetrafluoroetileno (Kaufman II) o prótesis de gel de silicona (Kaufman III) ${ }^{1}$. En estas técnicas, los puntos de fijación se realizan en tejidos blandos, que con el tiempo pierden su consistencia, disminuyendo por tanto la resistencia uretral artificialmente creada y facilitando así la recidiva de la incontinencia. Por el contrario, la fijación ósea del Invance ${ }^{\circledR}$, permite una mayor estabilidad a lo largo del tiempo, disminuyendo la probabilidad de recidiva (Tabla 4).

Durante la década de los 90 aparecen técnicas de sling, las cuales utilizan diversos materiales: fascias autólogas, material cadavérico y slings sintéticos. Estos materiales fueron inicialmente aplicados con fijación a la fascia de los rectos abdominales. Schaeffer ${ }^{3}$, Migliari ${ }^{4}$ e Hubert John ${ }^{5}$ presentaron series con slings, cuya red era fijada en la región supra-púbica, sobre la fascia de los rectos abdominales, siendo por tanto necesaria una incisión abdominal y el paso de los hilos de Prolene $^{\circledR}$ a través del espacio retropúbico con la ayuda de agujas de Stamey. Con la técnica de Invance $^{\circledR}$, la red es fijada en el ramo isquiopúbico, por lo que no es necesaria la incisión abdominal, como tampoco la invasión del espacio retropúbico (maniobra ésta con un elevado po-

\section{Tabla 4}

IUE masculina - Sling

Kaufman (1970)

Puigvert (1971)

Salcedo (1972)

Servadio (1974)

Yarbrough (1975)

Jorion (1997)

Mizuo(1992)

Schäefer (1998)

$\operatorname{Raz}$ (1998)

Cespedes (1999)

Franco (2001) tencial iatrogénico, vistas las eventuales secuelas resultantes de la cirugía abdominal previa y/o la radioterapia post-operatoria, presentes en muchos de estos pacientes) ${ }^{3}$. Es una técnica relativamente reciente y como tal no existen actualmente series con un número suficiente de pacientes para realizar conclusiones estadísticas, que permitan compararla con técnicas anteriores, como el esfinter urinario artificial. Los pacientes seleccionados para esta técnica debe ser cuidadosamente evaluados a través de la historia clínica, examen físico, estudio urodinámico y cistoscopia. Los resultados clínicos iniciales refieren tasas de curación entre los $80 \%^{2}$ y $85,7 \%^{3}$, después de 12 meses de seguimiento (Tabla 5).

Los pacientes con vejigas hipocontráctiles no son los candidatos ideales para la colocación del esfinter artificial, por existir un elevado peligro de ser necesaria la auto-cateterismo durante el post-operatorio, este peligro debe ser ponderado y discutido con el paciente. A pesar de que esfinter urinario artificial pueda continuar siendo el tratamiento de referencia para estos pacientes, la aplicación del sling no está contra-indicada, ya puede igualmente resolver la incontinencia. En el caso de no tener éxito, la aplicación del sling no invalida la posterior colocación del esfínter artificial ${ }^{3}$.

Colocamos un sling en un paciente con neovejiga tras explicarle los riesgos. El paciente era portador de una malformación de las manos que le impediría la manipulación del esfinter artificial, por lo cual y después de nuestra explicación, el paciente aceptó la colocación del sling.

El peligro de erosión de la uretra, situación temida en otras técnicas, es menos importante en el implante del sling. En esta técnica la uretra es apenas comprimida en su cara inferior, lo que permite una buena vascularización, tanto de la uretra como de los tejidos peri-uretrales. La mayor tasa de erosión descrita es con el esfinter urinario artificial; ésto puede ser el resultado de la presión ejercida sobre la uretra, la cual es comprimida en forma circunferencial, y solamente se ve aliviada durante a micción ${ }^{5}$. No fue referido ningún caso de erosión en los trabajos publicados hasta el momento. El riesgo de complicaciones óseas, como la osteomielitis fue descrito en slings con fijación ósea, usados en la incon- 


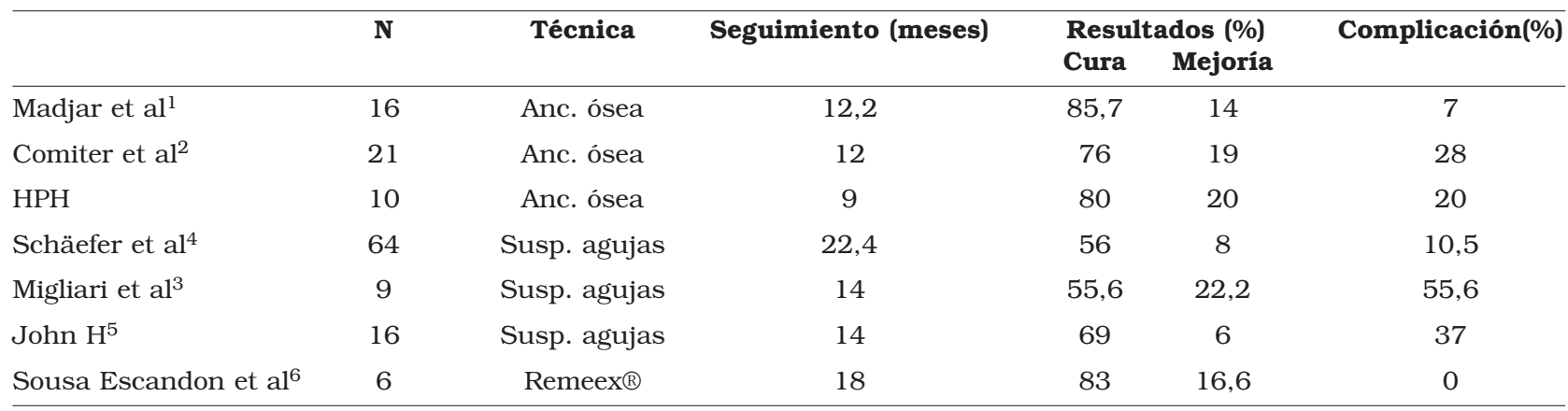

tinencia femenina. En estos casos la fijación de la red es hecha a través de la vagina, pudiendo haber contacto del material con la flora bacteriana vaginal, aumentando así el peligro de infecciones. En el hombre, la fijación se realiza en un campo quirúrgico estéril, reduciéndose así el peligro de contaminación y la posibilidad de complicaciones infeciosas ${ }^{3}$. Los resultados clínicos presentados en nuestra serie son prometedores y están de acuerdo con otras series. El dolor perineal fue referido en $20 \%$ de nuestros pacientes, pero igualmente fue referido en otras séries ${ }^{3}$. Es un dolor de corta duración y que cede fácilmente con la toma de paracetamol. En nuestra serie no se verificaron hematomas, infecciones, rechazo del material $u$ otras complicaciones. Los dos pacientes con pérdidas urinarias, presentan una incontinencia ligera, la cual que no tiene impacto en sus actividades diarias.

Por último este sling es menos costoso que el esfinter urinario artificial, estando calculado su valor aproximado, en cerca de $77 \%$ menos que el esfinter ${ }^{2}$.

\section{CONCLUSIÓN}

La colocación del sling con fijación en el hueso púbico es una técnica de fácil ejecución. No necesita de la colaboración del paciente para su funcionamiento. Las complicaciones post-opera- torias son raras y resulta una técnica más económica comparada con otros métodos terapéuticos.

A pesar de ser necesario un seguimiento más largo, los datos ya obtenidos por nosotros, nos hacen pensar que se trata de una técnica segura y eficaz a explorar en un futuro próximo.

\section{REFERENCIAS}

1. S. Madjar, K. Jacoby, C. Giberti, et al. Bone anchored sling for the treatment of post-prostatectomy incontinence. J Urol 2001;165:72-76.

2. V. Comiter. The male sling for the stress urinary incontinence: a prospective study. J Urol 2002;167:597-601.

3. A. Schaeffer, J. Clemens, M. Ferrari, T. Stamey. The male boulbourethral sling for post-radical prostatectomy incontinence. J Urol 1998;159:1510-1515.

4. R. Migliari, D. Pistolesi, M. De Angelis. Polypropilene sling of the bulbar urethra for post-radical prostatectomy incontinence. Euro Urol 2003;43:152-157.

5. H. John. Boulbourethral composite suspension: a new operative technique for post-prostatectomy incontinence. J Urol 2004;171:1866-1870.

6. A. Sousa-Escandón, J.I.R. Gómez, C.U. González, A.M. Queimadelos. Externally readjustable sling for treatment of male stress urinary incontinence: points of technique and preliminary results. J Endo Urol 2004;18(1):113-118.

Dr. M. Cerqueira

Rua Elaine Sanceau, $942^{\circ}$ esq.

4465 Sao Mamede de Infesta

Portugal

(Trabajo recibido el 1 diciembre de 2004) 